\title{
The value of PET, CT and in-line PET/CT in patients with gastrointestinal stromal tumours: long-term outcome of treatment with imatinib mesylate
}

\author{
G. W. Goerres ${ }^{1}$, R. Stupp², G. Barghouth ${ }^{3}$, T. F. Hany ${ }^{1}$, B. Pestalozzi ${ }^{4}$, E. Dizendorf1, 5, P. Schnyder ${ }^{3}$, F. Luthi², \\ G. K. von Schulthess ${ }^{1}$, S. Leyvraz ${ }^{2}$ \\ 1 Division of Nuclear Medicine, University Hospital Zurich, Zurich, Switzerland \\ 2 Multidisciplinary Oncology Centre, University of Lausanne Medical Centre, Lausanne, Switzerland \\ ${ }^{3}$ Department of Radiology, University of Lausanne Medical Centre, Lausanne, Switzerland \\ 4 Department of Oncology, University Hospital Zurich, Zurich, Switzerland \\ 5 International Tomography Center, Novosibirsk, Russia
}

Received: 15 January 2004 / Accepted: 16 June 2004 / Published online: 4 September 2004

(C) Springer-Verlag 2004

\begin{abstract}
Purpose: Gastrointestinal stromal tumours (GIST) are mesenchymal neoplasms of the gastrointestinal tract that are unresponsive to standard sarcoma chemotherapy. Imaging of GIST patients is done with structural and functional methods such as contrast-enhanced helical computed tomography (ceCT) and positron emission tomography (PET) with ${ }^{18} \mathrm{~F}$-fluorodeoxyglucose (FDG). The aim of this study was to compare the prognostic power of PET and ceCT and to evaluate the clinical role of PET/CT imaging. Methods: All patients with GIST undergoing PET or PET/CT examinations were prospectively included in this study, and the median overall survival, time to progression and treatment duration were documented. The prognostic significance of $\mathrm{PET}$ and ceCT criteria of treatment response was assessed and PET/CT was compared with PET and ceCT imaging. Data for 34 patients (19 male, 15 female, 2176 years) undergoing PET or PET/CT for staging or restaging were analysed. Results: In 28 patients, PET/CT and ceCT were available after introduction of treatment with the tyrosine kinase inhibitor imatinib mesylate (Gleevec; Novartis, Basel, Switzerland). Patients without FDG uptake after the start of treatment had a better prognosis than patients with residual activity. In contrast, ceCT criteria provided insufficient prognostic power. However, more lesions were found on ceCT images than on PET images, and FDG uptake was sometimes very variable. PET/CT delineated active lesions better than did the combination of PET and ceCT imaging. Conclusion: Both PET and PET/CT provide important
\end{abstract}

G. W. Goerres (®)

Division of Nuclear Medicine, University Hospital Zurich,

Raemistrasse 100, 8091 Zurich, Switzerland

e-mail: gerhard.goerres@usz.ch

Tel.: +41-1-2552850, Fax: +41-1-2554414 prognostic information and have an impact on clinical decision-making in GIST patients. PET/CT precisely delineates lesions and thus allows for the correct planning of surgical interventions.

Keywords: GIST - Treatment response - Molecular imaging - FDG PET - Image co-registration

Eur J Nucl Med Mol Imaging (2005) 32:153-162

DOI 10.1007/s00259-004-1633-7

\section{Introduction}

Gastrointestinal stromal tumours (GIST) are mesenchymal tumours that in approximately $90 \%$ of cases originate in the stomach and small intestine [1]. They have a wide clinical spectrum ranging from benign incidentally detected nodules to large malignant tumours and must be distinguished from other mesenchymal tumours [2]. There is a range of histological variants. GIST are defined as KIT-positive tumours because they have a common molecular pathogenesis with activating mutations in the gene encoding KIT (CD117, stem cell factor receptor) and positivity of cKIT on immunohistochemistry is a conditio sine qua non for the diagnosis of GIST [2]. Coexpression of KIT with CD34 can often be found since both originate from a CD34-positive subset of Cajal cells, the pacemaker cells of the gastrointestinal tract $[2,3]$.

The only curative therapy for localised GIST is surgery. Tumours are usually resistant to conventional cytotoxic chemotherapy and radiation [4]. Recently a targeted therapy with imatinib mesylate (also referred to as STI-571, Gleevec or Glivec; Novartis, Basel, Switzerland), a receptor tyrosine kinase inhibitor, has been intro- 
duced for the treatment of GIST. This substance inhibits the activated KIT protein located on the tumour cell membrane [5].

Structural imaging with contrast-enhanced helical computed tomography (ceCT) is widely used to assess therapy response in GIST patients [6]. It has previously been shown that GIST tumours treated with imatinib mesylate may not change their size or may even grow larger during therapy [7]. However, the assessment of tumours responding to treatment with imatinib mesylate may show a decrease in CT attenuation values (Hounsfield units, HU) [7]. A delay of several weeks to months can be observed between the functional changes at a cellular level that are induced by an effective treatment and the macroscopic structural changes in a tumour as measured by ceCT [8]. In contrast, positron emission tomography (PET) using ${ }^{18} \mathrm{~F}$-fluorodeoxyglucose (FDG), a glucose analogue which is taken up into cells without being further metabolised, is able to show early effects in patients undergoing treatment with imatinib mesylate $[8,9]$. Additionally, recent studies have suggested that a decrease in FDG uptake after the start of imatinib mesylate treatment indicates a positive treatment result and a prolonged progression-free survival $[8,10]$.

The first aim of this study was to compare the prognostic value of early PET imaging after introduction of imatinib mesylate treatment with the prognostic value of ceCT imaging. We correlated imaging response with time to progression and overall survival. Secondly, the findings of PET imaging were compared with those of ceCT and the results of in-line PET/CT were compared with the combined reading of PET and ceCT images in order to assess the additional value of PET and PET/CT imaging.

\section{Materials and methods}

\section{Patients}

Consecutive patients with histologically proven CD117-positive GIST undergoing PET and PET/CT examinations in our institution were analysed. All patients were treated with imatinib mesylate. The patients were followed prospectively with clinical examinations and radiological imaging every 3 months. Patients received either a 400- or an 800-mg starting dose of imatinib mesylate per os daily. In the event of progression at $400 \mathrm{mg}$, the dose was increased to $800 \mathrm{mg} /$ day. In cases of toxicity, the doses were reduced. The local ethics committee approved the protocol and all patients gave informed written consent for participation in the study.

\section{Imaging}

PET imaging was performed on an Advance and a Discovery LS camera (both GE Medical Systems, Waukesha, WI). In the Discovery LS, an Advance NXi PET camera and a multislice helical CT scanner (LightSpeed plus) are integrated to allow the acquisition of automatically aligned CT and PET images in one session. Patients fasted for at least $4 \mathrm{~h}$ prior to scanning, which was started approximately $45 \mathrm{~min}$ after the injection of a standard dose of approximately $370 \mathrm{MBq}$ of FDG. The supine patients were examined if possible with the arms above the head. First, the CT scan was acquired starting at the level of the head and using the following parameters: $80-\mathrm{mA}$ tube current, $0.5 \mathrm{~s}$ per tube rotation, $140-\mathrm{kV}$ tube voltage, slice thickness of $5 \mathrm{~mm}$ with four simultaneous slice acquisitions and a helical pitch of approximately six over a $867-\mathrm{mm}$ scan length covering the body from the head to the pelvic floor. This whole-body CT scan was acquired in $22.5 \mathrm{~s}$ and in a breath-hold respiration position to avoid artefacts due to erroneous image co-registration and attenuation correction [11]. In all patients, oral contrast medium was given for $1 \mathrm{~h}$ starting approximately 60 min before scanning to improve the delineation of intestinal organs [12]. However, the CT scan was done without intravenous contrast enhancement, because all patients had already undergone ceCT as part of their routine work-up. Immediately following the $\mathrm{CT}$ acquisition, the PET emission scan was acquired starting at the pelvic floor. In both scanners the PET camera has a 14.6- $\mathrm{cm}$ axial field of view and a transaxial resolution of $4.8 \mathrm{~mm}$ full-width at half-maximum at the centre. The PET scans were obtained using an acquisition time of $4 \mathrm{~min}$ for the emission data. On the combined PET/CT scanner the CT data were used for attenuation correction, thus reducing the examination time to approximately $25 \mathrm{~min}$. On the Advance PET camera a conventional transmission scan of 2 min per cradle position, with a one-slice overlap at the borders of the field of view, was acquired. In contrast, scanning a patient using six table positions covering the body from the pelvic floor to the head resulted in an acquisition time of roughly $36 \mathrm{~min}$. During image acquisition the patients were under supervision by a technician. The PET data were reconstructed using a standard iterative algorithm (OSEM, 28 subsets, 2 iterative steps). The decision on whether to perform PET or integrated PET/CT depended on the availability of the scanner, except in a subgroup of patients who underwent PET/CT scanning before and after the introduction of imatinib mesylate treatment.

In these patients the helical ceCT was acquired in the university hospital of Lausanne using a LightSpeed Ultra eight-detector CT (General Electric Medical Systems, Waukesha, WI). Helical CT was obtained with section collimation of $5 \mathrm{~mm}$ with a pitch of 1.675:0.8. Scanning of the chest and abdomen was performed after initiation of contrast infusion, at $25 \mathrm{~s}$ for thorax and at $60 \mathrm{~s}$ for abdomen. Oral administration of water or contrast (2\%) was given 20 min before examination and contrast enema (2\%) was given immediately before examination. The contrast enhancement was obtained with $80 \mathrm{ml}$ of iodinated material (Accupaque 300) injected with a mechanical power injector at a rate of $2.7 \mathrm{ml} / \mathrm{s}$.

Image analysis

The acquired images were viewed with software providing multiplanar reformatted images of PET, CT and fused data with linked cursors (eNtegra GE Medical Systems, Haifa, Israel). PET and CT images were displayed using black and white images. The co-registered PET/CT scans were displayed using a standard grey scale for the CT images and a colour scale for the PET data. The CT images were viewed using soft tissue, lung and bone window image levelling. More than one reader visually assessed PET, ceCT and PET/CT images, and a consensus reached.

In the subset of patients who underwent PET/CT before and after treatment onset, the transverse images of the ceCT examination before and after contrast enhancement were developed on film. Two nuclear medicine physicians and two radiologists read 
the PET, co-registered PET/CT and ceCT images of each patient in consensus. The anatomical location of FDG-avid lesions on PET/CT images was directly compared with that on the ceCT images. Thus, the measurement of FDG concentrations and HU was done for identical lesions. Images were assessed visually and in addition the following parameters were measured: HU before and at the first control, size of reference lesions, the distribution pattern of FDG uptake and the visual intensity of FDG uptake before and during treatment. The visual intensity of a lesion was classified as high, intermediate or low when, respectively, FDG uptake was comparable to brain uptake, less than brain uptake but more than uptake in normal liver tissue or not discernible from normal soft tissue activity.

Response on ceCT was assessed according to the RECIST criteria (response evaluation criteria in solid tumours) used for therapy monitoring of malignant diseases [13]. In the control examination a suspicious finding on ceCT was either a new lesion or an increase in lesion size compared with previous examinations. FDG uptake was measured in regions of interest (ROIs) drawn on the PET image, and the mean value of the ROI was taken to calculate standardised uptake values (SUVs). The ROI was defined on the transverse slice of the corresponding CT image of the co-registered PET/CT scan where the lesion had the largest transverse diameter. The mean SUV was corrected for the body weight of the patient. The same lesions were used as a reference to assess therapy response on ceCT images. All measurements were done by one experienced physician using PMOD software (ED; PMOD Version 2.35; www.pmod.com).

On the PET images the treatment effect was measured according to the recommendations of the EORTC PET study group [14]. Because in GIST tumours it is not uncommon to find several lesions, a complete remission was defined as the disappearance of all FDGavid lesions. In addition to the SUV criterion defined by the EORTC PET study group, we defined a partial response as a decrease in the number of visible lesions with or without a decrease in FDG uptake or a decrease in FDG uptake with or without a reduction in the number of lesions. Progression was defined as the appearance of new lesions or an increase in the SUV of the target lesion by $>25 \%$. Stable disease was defined as an increase in FDG uptake of $<25 \%$ or a decrease of $<15 \%$ and a stable number of FDG-avid lesions [14]. If a patient did not have visually increased uptake on the pre-treatment PET and was still negative at the first control examination, the patient was categorised as having stable disease.

Subsequently a separate evaluation of the PET/CT and ceCT scans was done before and after the introduction of treatment with imatinib mesylate: two readers retrospectively assessed the ceCT with the corresponding PET images of a patient and compared the findings with the co-registered PET/CT images of the same patient. Both examinations were read in consensus to evaluate the possible advantage of PET/CT image co-registration over ceCT with separate PET images for the anatomical delineation of active lesions.

\section{Statistical evaluation}

All values are given as mean \pm standard deviation. The relative decreases in FDG uptake and CT HU as measured in an ROI of patients with either stable disease or partial or complete response are given as percentage values. The time-to-event variables were analysed with the log rank test and displayed on Kaplan-Meier plots according to the imaging method used. The hazard ratios and 95\% CI were estimated. Overall survival, time to progression and time on medication with imatinib mesylate were calculated ac- cording to the Kaplan-Meier method from the first day of treatment. Survival comparisons were expressed using the log rank test. All statistical analyses were done using the SPSS software, version 10.0 for Mac.

\section{Results}

The imaging and clinical data of 34 consecutive patients were available for this study (19 male, 15 female, mean age 51.7 \pm 15.2 years, age range $21-76$ years) (Table 1 ). A total of 78 PET or PET/CT scans were acquired in these patients for staging and restaging and during follow-up. All patients except nos. 22, 26 and 27 underwent one or several surgical interventions before the PET or PET/CT examination (Table 1).

The median overall survival of the patients was 44.5 months with a 2-year survival of $78 \%(95 \%$ $\mathrm{CI}=63-94 \%)$. The median time on treatment with imatinib mesylate was also 44.5 months, with $67 \%$ of patients still taking imatinib mesylate after 2 years (95\% CI $=49-85 \%)$. The median time to progression was 23.5 months (range 13.4-33.6 months), with a 2-year progression-free survival of $49 \%(95 \% \mathrm{CI}=28-69 \%)$.

\section{Imaging findings of PET and PET/CT in 34 patients}

FDG-avid lesions were found in the liver ( $n=16$ patients; one to seven lesions/patient) and the gastrointestinal tract with or without extension into the mesentery or omentum and the retroperitoneal soft tissues ( $n=20$ patients; one to five lesions/patient) (Table 1). An example of PET/CT findings in a patient with abdominal GIST before treatment (patient 10) is given in Fig. 1. Extra-abdominal tumour involvement was found in mediastinal lymph nodes, oesophagus, axillary lymph nodes, lung and bones ( $n=4$ patients; one to seven extra-abdominal lesions/patient) and confirmed by either endoscopy or fine-needle aspiration with cytology (patients 3,12 and 32; Fig. 2).

FDG PET identified more skeletal lesions than ceCT images (in patients 14 and 32, involvement of a rib was missed on ceCT). In seven patients no FDG-avid lesions were found (patients 16, 17, 19, 21, 24, 33 and 34). In patients 5 and 14, liver lesions were suspected on the CT scan, but FDG uptake was not increased on the PET images (i.e. the uptake did not exceed physiological uptake in the adjacent normal liver tissue); however, in the same patients other liver lesions did show an increased FDG uptake (Table 1). In patient 11 , a lesion at the inferior pole of the kidney was visible on the PET image, but was believed to be a part of the urinary collecting system. The combined information obtained using ceCT with PET and co-registered PET/CT revealed the presence of the tumour. In patient 15 , three pulmonary lesions each smaller than $5 \mathrm{~mm}$ were missed on the PET images, but were correctly identified on the CT scan of combined PET/CT. 
Table 1. Patient characteristics

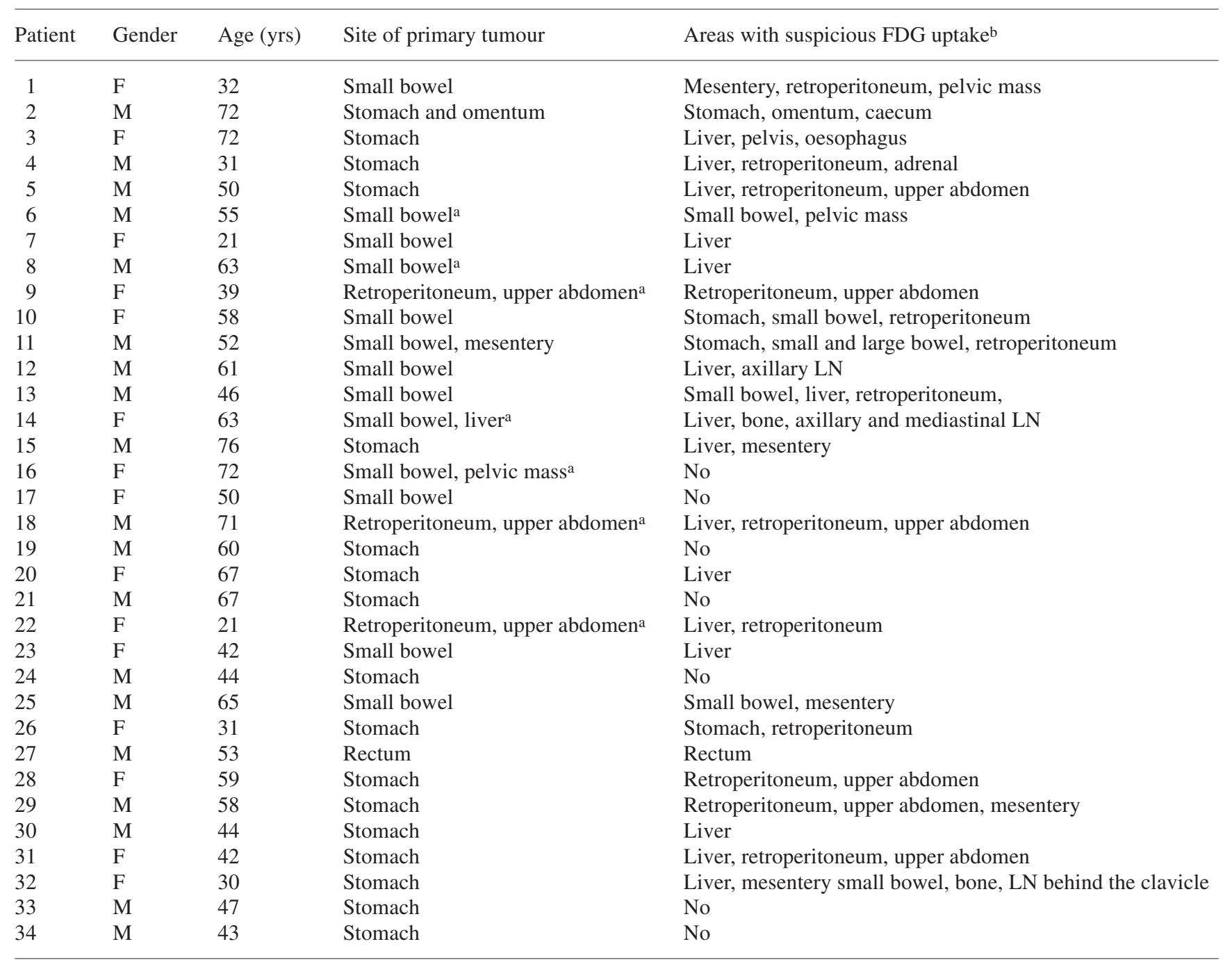

$L N$ lymph node

a The given location is probably the primary site

\section{Impact on clinical decision-making}

Indications for PET and PET/CT scanning were as follows: (a) for staging purposes, (b) to assess the effect, shortly after the initiation of treatment, of imatinib mesylate in patients who had had a pre-treatment PET or PET/CT scan, (c) to confirm a positive treatment effect in patients with a reduction in tumour size on the routine ceCT images during follow-up, (d) to confirm recurrent disease in patients with suspicious findings on routine ceCT images during follow-up, thus allowing adaptation of the dosage of imatinib mesylate, (e) to control at an early time point the efficacy of dosage adaptation in patients in whom dosage had been increased, (f) to control at an early time point the efficacy of a lower dosage in patients in whom dosage had been decreased, (g) to iden- b "No" indicates no increased FDG uptake visible at the time of chronologically first PET or PET/CT scan, i.e. FDG uptake not distinguishable from background activity or less than normal soft tissue activity

tify a (solitary) lesion with increased FDG uptake in a patient with otherwise stable disease for the planning of a selective surgical intervention and (h) to control the success of a surgical intervention.

For the assessment of surgical operability, one patient (no. 18) underwent PET scanning before salvage surgery. The same patient underwent PET/CT scanning to evaluate success of this intervention. Overall, three patients initially considered as inoperable underwent surgery with a curative intent after response to therapy with imatinib mesylate ( $8 \%$ of patients, $4 \%$ of all PET scans). In these three patients, PET and PET/CT information served to correctly delineate the extension of a known lesion by virtue of its increased FDG uptake and to rule out further lesions that might have been missed on the ceCT images. 

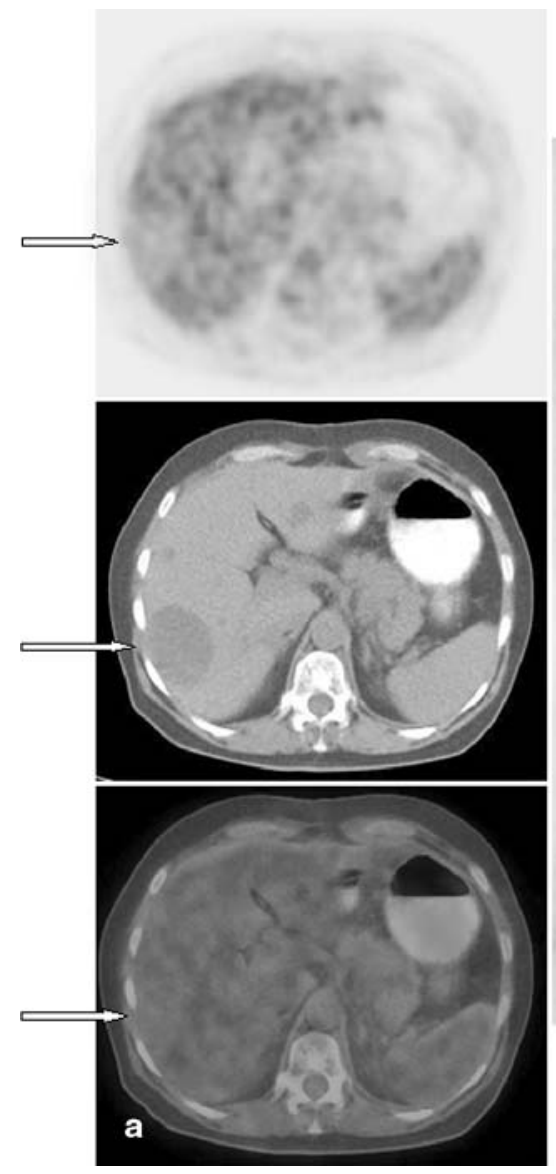

Fig. 1. a Coronal maximum intensity projection image (MIP) and transverse PET (top), CT (middle) and co-registered PET/CT images (bottom) of a 58-year-old female patient (patient 10) before the introduction of treatment with imatinib mesylate. On the MIP image, focal areas with increased FDG uptake are seen in the mid abdomen (long arrow) and at the oesophago-gastric junction (short arrow). An axillary lymph node is visible owing to paravenous tracer injection (asterisk). In the lower abdomen the appearance of bowel uptake is not suggestive of tumour involvement. In the transverse section at the level of the liver, no foci with increased tracer uptake are visible, but the largest of the three hypodense areas on the CT scan is also detectable on the FDG PET image as an area with low FDG uptake (open arrows). b On the transverse PET, CT and co-registered PET/CT images, areas with increased FDG uptake and corresponding soft tissue mass are present in the region of the duodenum, extending to the retroperitoneal soft tissues (large open white and black arrows), and at the anterior wall of the peritoneal cavity (long white and black arrows). There is a normal appearance of both kidneys and metal clips are visible after previous surgical intervention (asterisk). Within the large soft tissue mass in the duodenum only relatively small foci took up FDG. c A focal site of FDG uptake was also visible on the PET and PET/CT images at the level of the oesophago-gastric junction (black arrows). This site corresponded to a non-enlarged lymph node metastasis. On the ceCT scan (not shown) and on the CT image of the PET/CT scan, this lymph node was not delineated (black arrow)

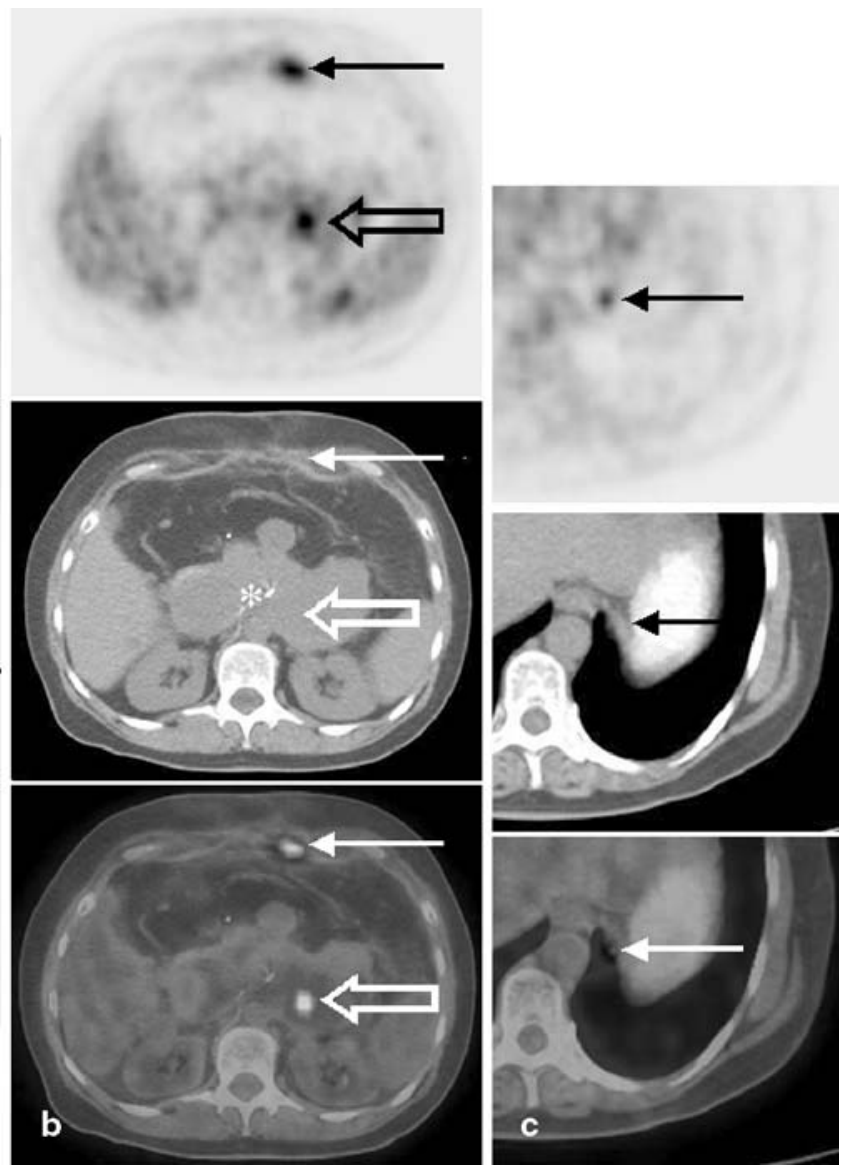

Comparison of PET/CT with PET and ceCT

In 15 of 34 patients a direct comparison of PET/CT findings with PET and ceCT findings was done. Before the start of therapy, 66 lesions were identified on the PET images of the PET/CT scan while 96 lesions were found on the ceCT images, i.e. $45 \%$ more lesions were seen with ceCT. The largest FDG-avid lesion ranged between $1 \mathrm{~cm}$ and $20 \mathrm{~cm}$ (mean $5.0 \pm 6.2 \mathrm{~cm}$, median $7.2 \mathrm{~cm}$ ) as measured on the corresponding CT image. In six patients, decreased FDG uptake was observed in the centre of the lesion, compatible with central necrosis. In small lesions the FDG uptake was usually strong and homogeneous, but in large lesions the distribution of FDG was often heterogeneous, showing areas with strong, intermediate, low or even no uptake (compatible with necrotic tissue).

The first follow-up ceCT examination in this subgroup showed signs of partial response $(n=6)$ or stable disease $(n=7)$ in 13 patients. In two patients, signs of progressive disease were found despite treatment with imatinib mesylate. The mean decrease in CT HU in the target lesion between staging and first follow-up ceCT in the six patients with a partial response was $39 \pm 22 \%$ (range 14-74\%). In the patients with stable disease, the HU did not decrease. In contrast, on the corresponding FDG PET images all patients showed complete remission $(n=9)$, partial response $(n=3)$ or stable disease $(n=3)$. In only four of 15 patients were concordant find- 


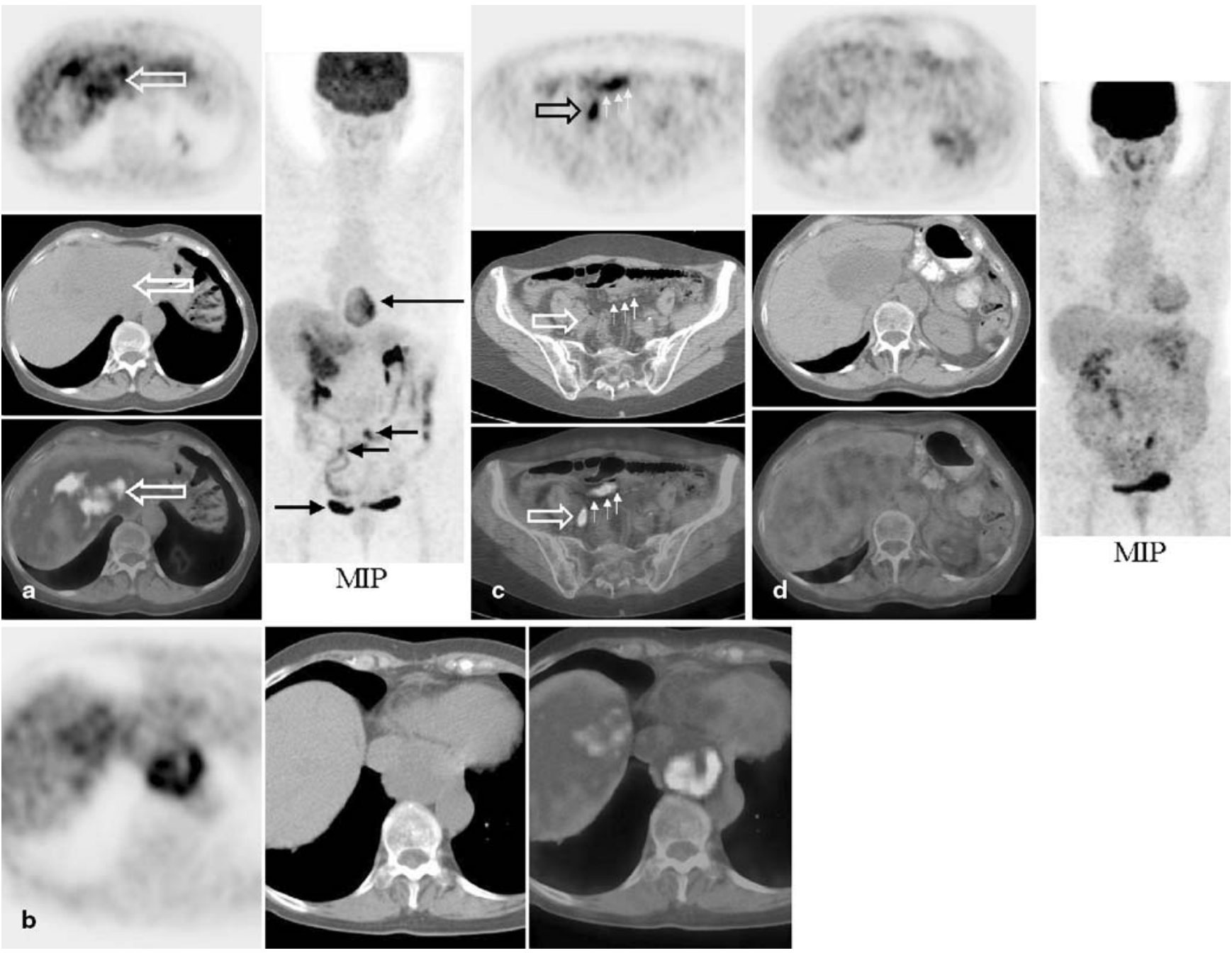

Fig. 2. a Coronal MIP image and transverse PET (top), CT (middle) and co-registered PET/CT images (bottom) of a 72-yearold female patient (patient 3 ) before the introduction of treatment with imatinib mesylate. A large FDG-avid and hypodense lesion is present in the liver, involving both lobes (white open arrows). FDG uptake in this large lesion is not homogeneous. On the MIP image a large lesion adjacent to the heart, which did not take up FDG, is visible (long black arrow). Smaller foci of increased tracer uptake are present in the middle and lower abdomen (small black arrows). b The lesion at the distal oesophagus shows intense FDG uptake (patient after total gastrectomy and splenectomy). c In the mid-abdomen and pelvis, several lesions were found corresponding to mesenteric lymph node involvement (white arrows) and uptake in the wall of non-enlarged bowel loops (white and black open arrows). The discrimination of such uptake from nonspecific muscular FDG uptake is difficult. d Seven weeks after the beginning of treatment a control scan was obtained, showing complete remission on the PET image and partial remission on the ceCT scan (not shown). On the CT image of the PET/CT scan, the liver lesion was less dense than on the first scan, but had not shrunk ings found on the first follow-up PET and ceCT, with either a partial response (patients 1 and 11) or stable disease (patients 13 and 15). The mean decrease in FDG uptake in the target lesion in patients with stable disease or a partial response was $55 \pm 20 \%$ (range $1-82 \%$ ). However, in patients with several lesions the decrease in FDG uptake in a target lesion did not necessarily reflect the behaviour of other lesions.

The evaluation of the additional value of co-registered PET/CT images over PET images read together with separate ceCT data revealed that in 25 of 30 examinations at least one of the lesions was better delineated on PET/CT images. In the other examinations PET with ceCT was judged to be equal to co-registered PET/CT in assessing the extent and size of a lesion. Furthermore, the two readers found that exact delineation of FDG-avid areas within large lesions would be useful to guide surgical procedures and to better assess operability.

\section{Prognostic information obtained by PET and CT}

In a subgroup of 28 patients $(82 \%, 15$ male, 13 female, mean age $52 \pm 15$ years, $21-76$ years) a PET or PET/CT 
Fig. 3. Overall patient survival according to the result of the first PET or PET/CT scan after treatment onset. Vertical axis, $\%$ of patients; horizontal axis, time. A Patients without suspicious FDG uptake on the image $(n=16)$ : the median survival was not reached. Mean 2-year survival was $100 \%$. $B$ Patients with suspicious FDG uptake on the image $(n=12)$ : the median survival was 22 months (range 9.1-38.5). Mean 2-year survival was $49 \%$ (range 20-52). There was a significant difference between these two patient groups $(\log$ rank test $p=0.001$ )

Fig. 4. Time to progression according to the result of the first post-treatment PET or PET/CT scan. Vertical axis, \% of patients; horizontal axis, time. $A$ Patients without suspicious findings on the PET image $(n=15)$ : the median time to progression was 32.2 months (range 23.1-41.3). The 2-year time to progression was $65 \%$ (range 41-90). B Patients with suspicious findings on the PET image $(n=9)$ : the median time to progression was 9.3 months (range 0-24.7). The 2-year time to progression was $22 \%$ (range 5-49). There was a significant difference between these two patient groups (log rank test $p=0.002$ )
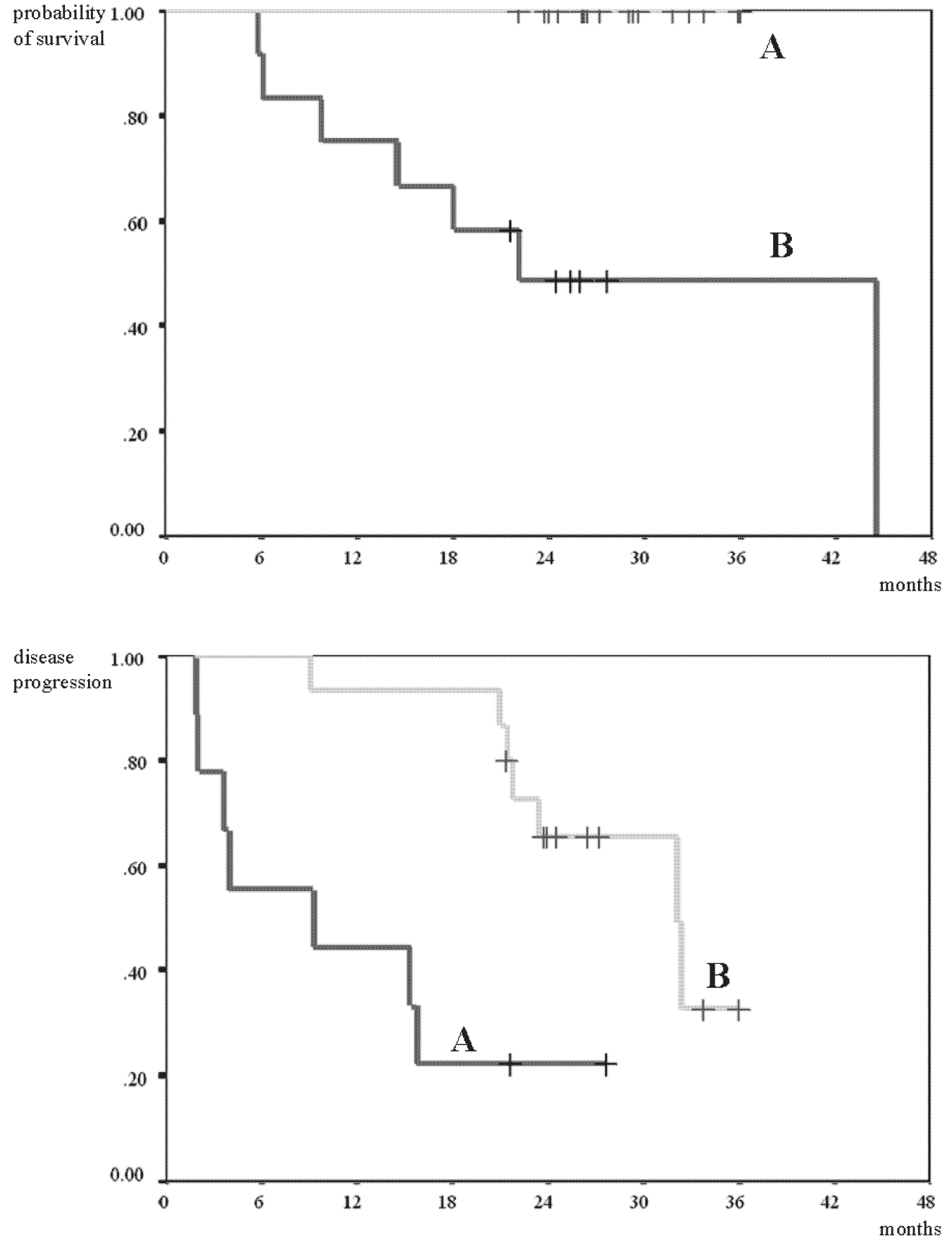

scan performed after the start of treatment was available, at a median interval of 19 days (range 11-111 days). The median overall survival of the 28 patients undergoing PET or PET/CT scanning after the start of imatinib mesylate treatment was 44.5 months, with a 2 -year survival of $80 \%(95 \% \mathrm{CI}=67-92 \%)$, indicating that this subgroup is comparable to the whole patient population. In 20 out of 28 patients a PET/CT scan was performed before and after treatment onset and, therefore, the response to imatinib mesylate treatment was measurable in these patients using both PET and CT criteria.

In the 28 patients in whom examinations were performed after treatment onset, the status of image criteria in the first follow-up examination and the change in PET and CT criteria between the pre-treatment and the first follow-up scan were used to compare the prognostic power of PET and CT. A post-treatment PET scan without pathological FDG accumulation was predictive of significantly better overall survival and longer time to progression compared with a follow-up PET image with FDG-avid areas ( $p=0.001$ and 0.002 , Figs. 3, 4). In contrast, CT findings were not suitable for prediction of overall survival or the time to progression, because in only two patients was the first follow-up CT scan considered to be normal ( $p=0.469$ and 0.030 ; log rank test $p=0.706)$. The measurement of changes between pre- 
treatment PET and first follow-up PET examination and between pre-treatment CT and first follow-up CT examination revealed a significant power of PET information in predicting the overall survival for responders (PET changes: log rank test $p=0.009$; CT changes: log rank test $p=0.706$ ). Measurement of changes between the pretreatment PET scan and the first follow-up PET examination was not suitable for discrimination of the time to progression in responders and non-responders (log rank test $p=0.129$ ). This was due to the inclusion of patients with FDG-negative scans before treatment onset in our evaluation: in such patients it was not possible to measure a decrease in FDG uptake between pre-treatment and post-treatment scans. Based on the CT criteria, again, no significant difference was observed for the time to progression between patients with and patients without a measurable change between the scans (log rank test $p=0.100$ ).

\section{Discussion}

The results of this study confirm previous reports showing a decrease in FDG uptake shortly after the introduction of treatment with imatinib mesylate [8-10]. We confirm other authors' findings that patients responding to treatment, as measured by normalisation of FDG-avid areas, have a better clinical outcome than patients in whom FDG uptake is still present [8-10]. In contrast to the short follow-up in previous reports, our patients have been followed up for a median of more than 3 years, and the minimum follow-up of the patients who are still alive is 22 months. Furthermore, in our study all PET examinations were considered, while in the study of Stroobants et al. patients with low FDG uptake at baseline were excluded [10]. In patients with negative PET scans before treatment onset, but with clinical evidence of active disease, comparative measurement of FDG activity between a pre-treatment and a post-treatment scan seems futile. However, our data suggest that a single post-treatment PET scan can provide prognostic information on overall survival and the time to progression, while a single posttreatment ceCT examination is not useful for this purpose.

More tumour lesions were found on ceCT images than on PET images. This may indicate that PET alone is insufficient to identify and characterise GIST lesions when surgery is intended. However, based on the longterm follow-up in our patients, it seems that FDG-avid lesions are clinically more important than lesions identified on ceCT images and CT images of the PET/CT scan, since they determine further management and clinical outcome. CT findings often remain equivocal in patients who have previously undergone surgery and who will show residual non-specific postoperative changes. Additionally, in most patients many lesions identified by CT cannot be confirmed histologically. Therefore, it is possible that reliance solely on the structural image information derived from ceCT will lead to false positive findings.

We found a broad spectrum of FDG uptake in the lesions. In large lesions a centrally decreased uptake was often seen, compatible with a necrotic area within the tumour. Furthermore, it was possible to find lesions with very high, moderate or no uptake at all in the same patient. This could have been due to the origination of lesions from different cell clones. GIST lesions can be missed on PET images, when pathological FDG uptake in a tumour cannot be discriminated from physiological uptake in the surrounding normal soft tissues. It is important to note that small FDG-avid lesions may be missed owing to the limited spatial resolution of the PET camera. Insufficient FDG uptake could have been the reason for the negative baseline PET in patient 21 with clinically active GIST. This patient was successfully treated with imatinib mesylate and remained FDG negative at two follow-up PET studies. This finding suggests that patients with clinically active disease may show no FDG-avid lesions before treatment onset, but nevertheless should be candidates for imatinib mesylate therapy.

Our data further suggest that co-registered PET/CT images facilitate the correct anatomical delineation of FDG uptake compared with the combination of separate ceCT and PET images. The examples in Figs. 1 and 2 illustrate how areas of increased FDG uptake can easily be co-registered to the anatomical structure as seen on CT images. In such cases the accuracy of PET/CT-based image co-registration could facilitate the identification of the optimal biopsy site and improve the planning of surgical procedures. In patients with equivocal ceCT findings after surgery, PET could identify active residual disease. Although to date it is not clear whether FDG-avid and non-avid areas differ in respect of histological properties, our data clearly suggest that information on FDG avidity has a clinical impact on further treatment decisions. Since FDG uptake in a lesion may reflect metabolic or even mitotic activity in GIST tumours, future studies should assess whether FDG uptake could serve as a direct measure of the aggressiveness of the tumour as defined by mitotic count [15]. Previous work has shown that FDG uptake is correlated with the amount of malignant cells or the aggressiveness of solid tumours [16, 17]. The assessment of operability is important since surgery with complete resection is the only curative therapy, and the prognosis of a patient with GIST (with low malignant potential) is excellent after complete resection [18]. Successful complete surgical resection is also important for the control of local recurrence or concomitant and metastatic disease [19]. Furthermore, it has recently been suggested that treatment with imatinib mesylate may improve the surgical outcome in patients with residual GIST tumour[20]. Therefore, in our patients many follow-up examinations were done to assess operability, 
and in three patients surgery was performed after PET/CT imaging. We found that the assessment of operability in GIST patients is facilitated by PET/CT. However, the clinical relevance of better anatomical delineation of FDG uptake remains to be demonstrated in future studies. Additionally, future studies will have to define clinical parameters suitable for indicating when follow-up examinations with PET or PET/CT should be performed and to determine whether routine control examinations at predefined time intervals are suitable for follow-up.

From a clinical point of view, stable disease under imatinib mesylate therapy signifies treatment success. In contrast, using RECIST and EORTC criteria for the assessment of CT and PET images only a measurable improvement, such as a decrease in lesion size or FDG uptake, is considered a response and indicative of successful therapy. We found it difficult to apply these imaging criteria to our GIST patients. If, for example, FDG uptake appeared in new lesions during follow-up while disappearing in old lesions, such a finding was still considered to indicate stable disease as long as the FDG uptake in the reference lesion remained stable. Because a single GIST patient can have many lesions, a lesionbased evaluation and direct comparison with CT images becomes utterly impossible. Therefore, the development of parameters which are easily applicable in routine imaging should improve the assessment of PET and PET/CT studies in this patient group.

In summary, the results of this study suggest that PET and PET/CT scanning have high prognostic relevance and yield clinically relevant information for treatment planning in GIST patients. This study also suggests that the use of a single post-treatment PET scan to identify the presence of FDG uptake is sufficient for the prediction of patient outcome and that a pre-treatment scan is not needed to predict overall survival. Patients not showing a response on a PET scan days to weeks after the start of treatment carry a worse prognosis and alternative treatment strategies should be considered. PET is essential to early treatment response assessment and CT imaging is needed to plan surgical interventions. These complementary methods are integrated in PET/ $\mathrm{CT}$, which was found to provide additional information compared with evaluation of separate PET and ceCT images.

\section{References}

1. Miettinen M, Sarlomo-Rikala M, Lasota J. Gastrointestinal stromal tumors: recent advances in understanding of their biology. Hum Pathol 1999;30:1213-20.

2. Miettinen M, Lasota J. Gastrointestinal stromal tumorsdefinition, clinical, histological, immunohistochemical, and molecular genetic features and differential diagnosis. Virchows Arch 2001;438:1-12.
3. Wang X, Mori I, Tang W, Utsunomiya H, Nakamura M, Nakamura Y, et al. Gastrointestinal stromal tumors: are they of Cajal cell origin? Exp Mol Pathol 2002;72:172-7.

4. Plaat BE, Hollema H, Molenaar WM, Torn Broers GH, Pijpe J, Mastik MF, et al. Soft tissue leiomyosarcomas and malignant gastrointestinal stromal tumors: differences in clinical outcome and expression of multidrug resistance proteins. J Clin Oncol 2000;18:3211-20.

5. Heinrich MC, Rubin BP, Longley BJ, Fletcher JA. Biology and genetic aspects of gastrointestinal stromal tumors: KIT activation and cytogenetic alterations. Hum Pathol 2002; 33:484-95.

6. Burkill GJ, Badran M, Al-Muderis O, Meirion Thomas J, Judson IR, Fisher C, Moskovic EC. Malignant gastrointestinal stromal tumor: distribution, imaging features, and pattern of metastatic spread. Radiology 2003;226:527-32.

7. Chen MY, Bechtold RE, Savage PD. Cystic changes in hepatic metastases from gastrointestinal stromal tumors (GISTs) treated with Gleevec (imatinib mesylate). Am J Roentgenol 2002; 179:1059-62.

8. Van den Abbeele AD, Badawi RD. Use of positron emission tomography in oncology and its potential role to assess response to imatinib mesylate therapy in gastrointestinal stromal tumors (GISTs). Eur J Cancer 2002;38(Suppl 5): S60-5.

9. Gayed I, Vu T, Iyer R, Johnson M, Macapinlac H, Swanston $\mathrm{N}$, Podoloff $\mathrm{D}$. The role of ${ }^{18} \mathrm{~F}-\mathrm{FDG}$ PET in staging and early prediction of response to therapy of recurrent gastrointestinal stromal tumors. J Nucl Med 2004;45:17-21.

10. Stroobants S, Goeminne J, Seegers M, Dimitrijevic S, Dupont $\mathrm{P}$, Nuyts J, et al. ${ }^{18}$ FDG-positron emission tomography for the early prediction of response in advanced soft tissue sarcoma treated with imatinib mesylate (Glivec). Eur J Cancer 2003; 39:2012-20.

11. Goerres GW, Kamel E, Heidelberg TN, Schwitter MR, Burger C, von Schulthess GK. PET-CT image co-registration in the thorax: influence of respiration. Eur J Nucl Med Mol Imaging 2002;29:351-60.

12. Dizendorf EV, Treyer V, Von Schulthess GK, Hany TF. Application of oral contrast media in coregistered positron emission tomography-CT. Am J Roentgenol 2002;179:477-81.

13. Therasse P, Arbuck SG, Eisenhauer EA, Wanders J, Kaplan RS, Rubinstein L, et al. New guidelines to evaluate the response to treatment in solid tumors. European Organization for Research and Treatment of Cancer, National Cancer Institute of the United States, National Cancer Institute of Canada. J Natl Cancer Inst 2000;92:205-16.

14. Young H, Baum R, Cremerius U, Herholz K, Hoekstra O, Lammertsma AA, et al. Measurement of clinical and subclinical tumour response using $\left.{ }^{18} \mathrm{~F}\right]$-fluorodeoxyglucose and positron emission tomography: review and EORTC recommendations. European Organization for Research and Treatment of Cancer (EORTC) PET Study Group. Eur J Cancer 1999; 35:1773-82.

15. Fletcher CD, Berman JJ, Corless C, Gorstein F, Lasota J, Longley BJ, et al. Diagnosis of gastrointestinal stromal tumors: a consensus approach. Hum Pathol 2002;33:45965.

16. Bos R, van Der Hoeven JJ, van Der Wall E, van Der Groep P, van Diest PJ, Comans EF, et al. Biologic correlates of (18)fluorodeoxyglucose uptake in human breast cancer measured by positron emission tomography. J Clin Oncol 2002; 20:379-87. 
17. Kitagawa Y, Sano K, Nishizawa S, Nakamura M, Ogasawara T, Sadato N, Yonekura Y. FDG-PET for prediction of tumour aggressiveness and response to intra-arterial chemotherapy and radiotherapy in head and neck cancer. Eur J Nucl Med Mol Imaging 2003;30:63-71.

18. Emory TS, O'Leary TJ. Prognosis and surveillance of gastrointestinal stromal/smooth muscle tumors. Ann Chir Gynaecol 1998;87:306-10.
19. DeMatteo RP, Lewis JJ, Leung D, Mudan SS, Woodruff JM, Brennan MF. Two hundred gastrointestinal stromal tumors: recurrence patterns and prognostic factors for survival. Ann Surg 2000;231:51-8.

20. Hohenberger P, Bauer S, Schneider U, Pinlk D, Dirsch O, Schuette J, Reichardt P. Tumor resection following imatinib pretreatment in GI stromal tumors. Proc Am Soc Clin Oncol 2003;22:818 (abstract). 